\title{
Is there any difference between Vitamin D deficiency among heart failure and non-heart failure elderly with cardiovascular disease?
}

\author{
Zohreh Taraghi $^{1}$, Ehteram sadat Ilali $^{{ }^{*}}$, Tahereh Yaghoobi ${ }^{1}$, Soheil Azizi ${ }^{2}$ \\ ${ }^{1}$ Faculty of Nursing and Midwifery, Mazandaran University of Medical Sciences, Sari, Iran \\ ${ }^{2}$ Faculty of Allied Medical Sciences, Mazandaran University of Medical Sciences, Sari, Iran
}

Received: Mar 27, 2016, Revised: Apr 6, 2016, Accepted: Apr 11, 2016

\begin{abstract}
Vitamin D deficiency is common among the elderly with and without heart failure (HF). This study was designed to identify the degree of vitamin D deficiency among HF and non- HF elderly with cardiovascular disease. In this study, fifty hospitalized elderly patients with HF (26 women and 24 men, age range 60-90 years) and fifty elderly without HF were randomly selected. The level of serum 25 -hydroxy vitamin D was measured using the Eliza method. There were no significantly difference between two groups regarding to the mean age, living location, life style parameters, mean years of disease involvement and disease severity, cardiovascular risk factors and comorbidities, except for renal problems $(p=0.039)$, hemoglobin levels $(p=0 / 044)$, and creatinine levels $(p=0.048)$. In both groups, the majority of patients, were moderately vitamin D deficient and there was no significantly difference between two groups.

Considering the high prevalence of vitamin D deficiency among HF and non-HF elderly with cardiovascular disease, its monitoring and correcting seems essential.
\end{abstract}

Keywords: Vitamin D deficiency, elderly, older people, heart failure, cardiovascular disease

Pharm Biomed Res 2016; 2(1): 36-43

DOI: 10.18869/acadpub.pbr.2.1.36

\section{Introduction}

In recent years, the pathophysiologic concept of heart failure (HF) has changed from an isolated hemodynamic view to more complex concept involving neurohormonal overactivation and increased concentration of proinflamatory cytokines, such as TNF and interleukin 6 $(1,2)$. Interestingly, experimental studies have shown that the vitamin $\mathrm{D}$ can suppress the release of TNF- $\alpha$ (2). Vitamin D deficiency seems to predispose to hypertension, diabetes and metabolic syndrome, left ventricular hypertrophy, chronic vascular inflammation, pathogenesis of atherogenic dyslipidemia and ischemic heart disease (3-5). Possible causal mechanism that may link vitamin D deficiency to heart failure, may involve the regulatory effect of $1,25(\mathrm{OH})_{2}$ Vit D on myocardial gen expressions (6).Vitamin D has also been shown to reduce the activation of the systemic and cardiac rennin angiotensin system, which contributes to hypertension and cardiac hypertrophy (4). 
Myocardial calcium homeostasis, which is crucial for the contractility and electrophysiology of the heart, is also partially regulated by $1,25(\mathrm{OH})_{2}$ Vit D. This is mediated by its influence on ion channels and enzymatic reactions (7). The results of several studies show that vitamin D supplementation significantly decreases all-cause mortality (6,8). Exposure of arms and legs for about 5 to 30 minutes ( depending of time of day, season, latitude, air pollution, the degree of cloud cover, age, the extent of clothing covering, and skin pigmentation) between the hours of $10 \mathrm{am}$ and $3 \mathrm{pm}$, twice a week is often adequate for conversion 7 dehydrocholestrol (existed in the skin) to pre-vitamin D3 (4). It has been demonstrated that regular exposure to UVB increases circulating $25(\mathrm{OH})$ Vit D above a level of $10 \mathrm{nmol} / \mathrm{l}(40 \mathrm{ng} / \mathrm{l})$ and also significantly reduces blood pressure by approximately $6 \mathrm{~mm} / \mathrm{Hg}$ in hypertensive patients (9). Sunlight exposure and dietary intake of vitamin D may not provide adequate levels of vitamin D. Some studies suggest an intake of at least $800 \mathrm{IU}$ of vitamin D daily among elderly, especially in the winter (10). Consumption of some drugs such as anticonvulsants, steroids, antiacids, $\mathrm{H} 2$ blockers and proton pomp inhibitors, prevent vitamin D absorption (11). According to several studies , 40 to $\% 100$ of US and European community dwelling elderly involved in vitamin D deficiency (6). Older people are prone to develop vitamin D deficiency, because of various risk factors including: decreased dietary intake, diminished sun light exposure, reduced skin thickness, impaired intestinal absorption, and impaired hydroxylation in the liver and kidneys (5,12). Vitamin D deficiency may play a significant role in the pathogenesis of heart failure $(6,7,13)$, though there is insufficient evidence to develop recommendations for strategies that improve vitamin $\mathrm{D}$ deficiency for HF patients. In addition, little is known about the degree of vitamin $\mathrm{D}$ deficiency among $\mathrm{HF}$ and non-HF elderly. The aim of this study was to identify the degree of vitamin D deficiency among HF and non-HF elderly with cardiovascular disease.

\section{Material and methods}

In this cross sectional study, fifty hospitalized elderly patients with HF and the same number elderly patients without HF were randomly selected. This study was conducted between October 2011 and March 2012, at the Sari Heart Center located in Mazandaran province in Iran. All non-HF patients had a diagnosis of ischemic heart disease. Their diagnosis were confirmed by the cardiologist and under angiography. Persons who had history of taking vitamin D during past six months, were excluded. Data gathering tool was a questionnaire that was developed by researchers and its validity and reliability were confirmed by expert panel and test - retest $(r=0.79)$.

In this questionnaire, there were some questions related to demographic characteristics (age, gender, location) and factors affecting vitamin $\mathrm{D}$ deficiency such as cardiovascular risk factors (history of diabetes, hyper-lipidemia, hypertension, overweight, anemia, hemoglobin level, ejection fraction $<40 \%$, mean of systolic and diastolic 
pressures, body mass index, disease severity (using the NYHA class), biochemical characteristics (fasting blood sugar, cholesterol, creatinine, phosphorus, calcium), the diseases and drugs predisposing vitamin D deficiency (history of hepatic, renal, and gastrointestinal diseases, consumption of sun-block, anticonvulsants, anti-acids, $\mathrm{H} 2$ blockers, steroids), environmental factors (the number of walks per week and its duration, the number of sun exposure per week and its duration, the number of fish consumption per week and the type of fish, the number of dairy product consumption per week). This study was approved by the Research Ethics Committee of the Mazandaran University of Medical Sciences (Number 90-3). After completing of the informed consent by participants, $5 \mathrm{~mL}$ of venous blood was taken. Sample centrifuging and serum extraction were done and were immediately frozen. The level of serum $25(\mathrm{OH})$ Vit D was measured by the Eliza method (IDS ltd kit, UK).

Although a consensus regarding the optimal level of serum $25(\mathrm{OH})$ Vit D has not yet been established, most experts define vitamin D deficiency as a $25(\mathrm{OH})$ Vit D level of $<50 \mathrm{nmol} / \mathrm{l}(19 \mathrm{ng} / \mathrm{ml})$ (14). Based on a randomized clustered sampling from the Tehran population that was designed by Endocrinology and Metabolism Research Center of Tehran University, the cut off point for vitamin D deficiency was considered less than 35 $\mathrm{nmol} / \mathrm{l}(15)$.

\section{Statistically analysis}

Logistic regression was performed for qualitative variables and T-test was used for quantitative variables. Values $p<0.05$ were considered significant. The results were analyzed by SPSS software version 16.

\section{Results}

Both HF and non-HF groups include 26 women and 24 men. The mean of the age were $73.2 \pm 7.69$ years and $70.44 \pm 7.8$ years in HF and non-HF patients, respectively $(\mathrm{p}=0.078)$. The majority of the elderly with HF (62\%) were rural, while the majority of non- HF elderly $(54 \%)$ were urban $(\mathrm{p}=0.16)$. The mean years of disease involvement were 5.06 \pm 4.7 years in HF patients (with CI 95\% 3.72-6.4) and $5.34 \pm 4.96$ years (with CI 95\% 3.93-6.75) in non-HF patients ( $\mathrm{p}=$ 0.77). Among HF patients, the number of elderly males with vitamin D deficiency were significantly more than females (83.3 versus $53.8 \%, p=0.026$ ).

The number of the patients with diabetes, hyperlipidemia, overweight, anemia and ejection fraction $(\mathrm{EF})<40 \%$ were more in the elderly with HF compared to those without. There was no significantly difference between two groups, except for EF ( $p<0.001)$ (Table 1). Regarding to severity of disease, the majority of $\mathrm{HF}(48 \%)$ and non- HF patients (40\%) were class IV $(p=0.559)$.

\section{Diseases and drugs}

HF patients had a more renal, hepatic, and gastrointestinal problems and a more consumption of $\mathrm{H}_{2}$ blockers and anticonvulsants drugs, compared to nonHF patients. The usage of sun-block, steroids, anti-acids, were more in non-HF patients compared to HF patients. There was no significantly difference between 
Table 1 Cardiovascular characteristics of the elderly with and without HF

\begin{tabular}{lllll}
\hline Variable & HF group & Non-HF group & sum & P \\
\hline Diabetes & $26(52 \%)$ & $22(44 \%)$ & $48(48 \%)$ & 0.548 \\
HLP & $19(38 \%)$ & $16(32 \%)$ & $35(35 \%)$ & 0.675 \\
BMI > 25 & $24(48 \%)$ & $16(32 \%)$ & $40(40 \%)$ & 0.153 \\
HTN & $28(56 \%)$ & $35(70 \%)$ & $63(63 \%)$ & 0.214 \\
Anemia & $32(64 \%)$ & $28(56 \%)$ & $60(60 \%)$ & 0.541 \\
EF<40\% & $34(68 \%)$ & $12(24 \%)$ & $6(46 \%)$ & $<0.001$ \\
\hline
\end{tabular}

HLP:Hyperlipidemia; BMI:Body Mass Index; HTN:Hypertension; EF:Ejection Fraction

two groups, except for renal problems ( $\mathrm{p}$ $=0.039$ ).

\section{Environmental factors}

The number of walks per week, its duration and sun exposure were lower in the elderly with $\mathrm{HF}$ compared to those without HF ( $\mathrm{p}=0.172$ and $\mathrm{p}=0.223$ and $p=0.426$ respectively). The amount of milk and fish consumption were more in the elderly with $\mathrm{HF}$ compared to those without HF ( $p=0.420$ and $p=0.546$ respectively).

\section{Biochemical characteristics, blood pressure and BMI}

The mean of two times fasting blood sugar, cholesterol, creatinine, and phosphor levels were more in the elderly with HF compared to those without HF. The mean of two times systolic and diastolic blood pressure during 3 days, BMI, hemoglobin, and calcium levels were lower in the elderly with $\mathrm{HF}$ compared to those without HF. There was significantly difference between two groups, regarding to hemoglobin and creatinine levels $(\mathrm{p}=0.044$ and $\mathrm{p}=0.048$ respectively).

\section{Vitamin D levels}

The mean vitamin D levels were $33.76 \pm$ 2.82 and $41.76 \pm 3.06 \mathrm{nmol} / \mathrm{l}$, in elderly persons with and without $\mathrm{HF}$ respectively $(\mathrm{p}=0.178)$. In both groups, the majority of patients $(38 \%, 19$ persons), were moderately vitamin D deficient (Table 2). The odds ratio of vitamin $\mathrm{D}$ deficiency was more in elderly with $\mathrm{HF}(\mathrm{OR}=1.35 ; 95 \%$ CI $0.87-2.21 ; \mathrm{p}$ $=0.151)$ compared to those without HF.

\section{Vitamin D deficiency group ( $<35 \mathrm{nmol} / \mathrm{l})$}

The odds ratio of the overweight was significantly more among HF patients compared to those without HF (OR = $1.73 ; 95 \%$ CI 1.22-2.68; $\mathrm{p}=0.031)$. In addition, the odds ratio of the gastrointestinal disorders $(\mathrm{OR}=1.81$; 95\% CI 1.23-2.68; $\mathrm{P}=0.022)$ and renal disorders $(\mathrm{OR}=1.74 ; 95 \% \mathrm{CI} 1.16-2.61$; $\mathrm{p}=0.031)$ were significantly more among HF patients compared to those without HF (Table 3). 
Table 2 Classification of vitamin D levels among elderly with and without HF.

\begin{tabular}{lcccccc}
\hline Vitamin D levels (nmol/l) & $<12.5$ & $12.5-25$ & $25-35$ & $35-50$ & $50-75$ & $>75$ \\
\hline HF & $7(14 \%)$ & $19(38 \%)$ & $8(16 \%)$ & $5(10 \%)$ & $8(16 \%)$ & $3(6 \%)$ \\
$50(100 \%)$ & & & & & & \\
Non HF & $2(4 \%)$ & $19(38 \%)$ & $6(12 \%)$ & $7(14 \%)$ & $10(20 \%)$ & $6(12 \%)$ \\
$50(100 \%)$ & & & & & & \\
Total & $9(9 \%)$ & $38(38 \%)$ & $14(14 \%)$ & $12(12 \%)$ & $18(18 \%)$ & $9(9 \%)$ \\
$100(100 \%)$ & & & & & & \\
\hline
\end{tabular}

Table 3 The odds ratio and 95\% confidence interval of precipitating factors of vitamin D deficiency, among elderly with HF in vitamin D deficiency group $(<35 \mathrm{nmole} / \mathrm{l})$.

\begin{tabular}{lcc}
\hline Variable & odds ratio $(95 \% \mathrm{CI})$ & $\mathrm{p}$ \\
Diabetes & $1.11(0.71-1.74)$ & 0.817 \\
$\mathrm{HTN}$ & $0.86(0.51-1.25)$ & 0.510 \\
$\mathrm{HLP}$ & $1.06(0.67-1.66)$ & 0.997 \\
BMI > 25 & $1.73(1.22-2.68)$ & 0.031 \\
Anemia & $0.99(0.63-1.56)$ & 0.821 \\
EF < 40\% & $2.61(1.32-3 / 53)$ & 0.002 \\
Renal problems & $1.74(1.16-2.61)$ & 0.031 \\
Hepatic problems & $1.37(0.74-2.54)$ & 0.436 \\
Intestinal problems & $1.81(1.23-2.68)$ & 0.022 \\
Sunblock & $0.43(0.07-2.39)$ & 0.451 \\
Anticonvulsant & $1.84(1.45-2.33)$ & 0.580 \\
Steroids & $0.58(0.11-2.94)$ & 0.839 \\
Antiacids & $1.39(0.9-2.15)$ & 0.302 \\
$\mathrm{H}_{2}$ blocker & $1.6(1.07-2.39)$ & 0.101 \\
\hline
\end{tabular}


The number of the elderly with HF who never walks per week, were more than those without HF $(41.2 \%$ versus $33.3 \%$; p $=0.397)$. The number of the elderly with HF who used sun exposure daily, were lower than those without HF $(55.6 \%$ versus $44.1 \%, \mathrm{p}=0.856$ ). There were no significantly differences between the number and type of fish usage, and the number of dairy consumption, among the elderly with and without HF ( $\mathrm{P}=0.241$ and $\mathrm{p}=1$ and $\mathrm{p}=0.194$ respectively).

\section{Discussion}

The results of this study didn't show significantly correlation between vitamin D levels among the elderly with and without HF. In both groups, there were no statistical relationship between the age and vitamin D deficiency. This finding is contrast with the results of some studies $(7,16)$ and adjusts with other ones $(17,18)$. With increasing of the age, the probability of vitamin D deficiency is increased, because of decreased skin thickness, decreased dietary intake, diminished physical activity out of home, increased prevalence of the kidney, liver and gastrointestinal problems (5). No significantly relationship between the age and vitamin $\mathrm{D}$ deficiency may be explained by the relative youth of participants (mean age 72). Among HF patients, the number of men with vitamin D deficiency were significantly more than women. This finding adjusts with the results of one study (19) and is contrast with other ones $(18,20)$. The reason may be a more disease severity among men compared to women. The odds ratio of the overweight was significantly more among HF patients compared to those without HF. Diminished exposure to UV light, associated with reduced outdoor activities and likely physical inactivity may account, in part, for the lower level of serum vitamin $\mathrm{D}$ in overweight participants (21). In addition, the lipid solubility of vitamin D modifies its bioavailability and may contribute to the lower level of serum vitamin $\mathrm{D}$ in overweight and obese participants (22,23). Among elderly with HF, there was not significantly relationship between the number of walks per week and vitamin $\mathrm{D}$ levels. It may be due to relatively small sample size. The odds ratio of the gastrointestinal and renal disorders were significantly more among the elderly with HF compared to those without HF. Vitamin D deficiency in chronic renal disease, may be due to hyper-phosphatemia. Hyperphosphatemia increases fibroblast growth factor 23, which decreases 25hydroxyvitamin D-1 $\alpha$-hydroxylase activity (24).

\section{Limitations}

A number of limitations exist with respect to the present study. Firstly, this is a cross sectional study and the correlations can not imply the causation relationships between parameters. Secondly, there are different normal vitamin $\mathrm{D}$ ranges for winter and summer in different countries. Thirdly, the sample size is relatively small. More ever, the data represent only the subjects who agreed to participate in this study.

\section{Conclusion}

Considering the high prevalence of vitamin D deficiency among the HF and 
Non- HF elderly with cardiovascular disease, its monitoring and correcting seems essential.

\section{References}

1. Rauchhaus M, Doehner W, Francis DP, Davos C, Kemp M, Liebenthal C, et al. Plasma cytokine parameters and mortality in patients with chronic heart failure. Circulation 2000;102:3060-7.

2. Schleithoff SS, Zittermann A, Tenderich G, Berthold HK, Stehle P, Koerfer R. Vitamin D supplementation improves cytokine profiles in patients with congestive heart failure: a double-blind, randomized placebo-controlled trial. Am J Clin Nutr 2006;83:754-9.

3. Guasch A, Bullo M, Rabassa A, Bonada A, Del Castillo D, Sabench F, et al. Plasma vitamin $\mathrm{D}$ and parathormone are associated with obesity and atherogenic dyslipidemia: a cross-sectional study. Cardiovasc Diabetol 2012;11:149.

4. Holick, MF. Vitamin D deficiency. N Engl J Med. 2007;357:266-81.

5. Watson KE, Abrolat ML, Malone LL, Hoeg JM, Doherty T, Detrano R, et al. Active serum vitamin $\mathrm{D}$ levels are inversely correlated with coronary calcification. Circulation 1997;96:1755-60.

6. Pilz S, Marz W, Wellnitz B, Seelhorst U, Fahrleitner-pammer A, Dimai HP, et al. Association of Vitamin D deficiency with heart failure and sudden cardiac death in a large cross-sectional study of patients referred for coronary angiography. J Clin Endocrinol Metab 2008;93:3927-3.

7. Green JJ, Robinson DA, Wilson GE, Simpson RU, Westfall MV. Calcitriol modulation of cardiac contractile performance via protein kinase C. J Mol Cell Cardiol 2006;41:350-9.

8. Autier P, Gandini S. Vitamin D supplementation and total mortality: a meta-analysis of randomized controlled trials. Arch Intern Med.2007;167;1730-7.

9. Pfeifer M, Begerow B, Minne HW, Natchtigall D, Hansen C. Effects of a short term vit D3 and calcium supplementation on blood pressure and parathyroid hormone levels in elderly woman. J Clin Endocrinol Metab 2001;86:1633-7.

10. Hanley DA, Shawn Davison K. Vitamin D deficiency in North America. J Nutrr 2005; 135:332-7.

\section{Conflict of interest}

None of the authors have financial or non-financial competing interests.

11. Vieth R.What is the optimal vitamin D status for health? Prog Biophys Mol Biol 2006;92:26-32.

12. Kimmelstiel C, Levine D, Perry K, Patel AR, Sadaniantz A, Gorham N, et al. Randomized, controlled evaluation of short- and long-term benefits of heart failure disease management within a diverse provider network: the SPAN-CHF trial. Circulation 2004;110:1450-5.

13. Zittermann A, Schleithoff SS, Tenderich G, Berthold HK, Koerfer R, Stehle P. Low vitamin D status: A contributing factor in the pathogenesis of congestive heart failure? J Am Coll Cardiol 2003;41:105-12.

14. 14-Bischoff-Ferrari HA, Giovannucci E, Willett WC, Dietrich T, Dawson-Hughes B. Estimation of optimal serum concentrations of 25-hydroxyvitamin D for multiple health outcomes. Am J Clin Nutr. 2006;84:18-28.

15. Hashemipour S, Larijani B, Adibi $\mathrm{H}$, Sedaghat M, Pajouhi M, Bastan-Hagh MH, et al."The status of biochemical parameters in varying degrees of vitamin $\mathrm{D}$ deficiency " J Bone Miner Metab 2006;24:213-8.

16. Nakamura $K$, Nishiwaki $T$, Ueno $K$, Yamamoto M. Age-related decrease in serum 25hydroxyvitamin D concentration in the frail elderly: a longitudinal study. J Bone Miner Metab 2007;25:232-6.

17. Kruavit A, Chailukit L, Thakinstian A, Sirphrapradang $\mathrm{CH}$, Rajatanavin R. Prevalence of vitamin D insufficiency and low bone mineral density in elderly Thai nursing home residents.BMC Geriatr 2012; 12:49.

18. Nashimoto $M$, Nakamura $K$, Matsuyama $S$, Hatakeyama M, Yamamoto M. Hypovitaminos $\mathrm{D}$ and hyperparathyroidism in physically inactive elderly Japanese living in nursing homes: Relationship with age, sunlight exposure and activities of daily living. Aging clin Exp Res 2002;14:5-12.

19. Ginter JK, Krithika S, Gozdzik A, Han Well H, Whiting S, Parra EJ. Vitamin D status of older adults of diverse ancestry living in the greater Toronto area.BMC Geriatr 2013;13:66.

20. Nakamura $K$, Nishiwaki $T$, Ueno $K$, Yamamoto M. Serum 25hydroxyvitamin D levels and activities of daily living in non 
institutionaized elderly japanese requiring care. J Bone Miner Metab 2005:23:488-94.

21. Need AG, Morris HA, Horowitz M, Nordin C. Effects of skin thickness, age, body fat, and sun light on serum 25 hydroxyvitamin D. Am J Clin Nutr 1993;58:882-5.

22. Parikh SJ, Edelman M, Uwaifo GL, Freedman RJ, Semega-Janneh M, Raynolds $\mathrm{J}$, et al. The relationship between obesity and serum 1,25-dihydroxyvitamin D concentrations in healthy adults. J Clin Endocrinol Metab 2004;89:1196-9.
23. Wortsman J, Matsuoka LY, Chen TC, Lu Z, Holick MF. Decreased bioavailability of vitamin $\mathrm{D}$ in obesity. Am J Clin Nutr 2000;72:690-3

24. Holick MF. Vitamin D for health and in chronic kidney disease. Semin dial 2005;18:266-75. 